\title{
A Procedure for Plasticity Error Correction for Determination of Residual Stresses by the ESPI-HD Method
}

\author{
Leonid Lobanov ${ }^{1, a}$, Frederico A.P. Fernandes ${ }^{2, b}$ and Viktor Savitsky ${ }^{1, c,{ }^{*}}$ \\ ${ }^{1}$ E.O.Paton Electric Welding Institute, Kyiv, Ukraine \\ ${ }^{2}$ Federal University of ABC, São Bernardo do Campo, 09606-045, Brazil \\ aholo@paton.kiev.ua, bcodoico@gmail.com, 'cviktor.savitsky@gmail.com
}

\begin{abstract}
Keywords: Residual Stresses, Speckle-Interferometry, Hole Drilling, ESPI-HD Method, Plasticity Effect
\end{abstract}

\begin{abstract}
The standard strain gage hole drilling method for residual stress determination assumes a linear elastic behavior of the material. However, if the stress level is high, plastic deformation may occur near the drilled hole, resulting in significant errors in the calculation of residual stress. The application of optical whole-field interferometry methods like speckle-interferometry (ESPI) for determining residual stresses allows to gather more information about the stress state of an object compared to the data from resistance strain gauges. Such advantage is achieved by calculating a significantly larger amount of displacement values induced by the hole-drilling. The ESPI-HD method additionally enables automated data analysis and selection of predefined areas with displacements in the vicinity of the drilled hole to determine the plasticity effect without the need of the material's yield stress. In the present work, an approach is described to determine the plasticity effect without using the yield stress. The approach is based on the fact that plastic deformation in the vicinity of the hole is not uniform. Analysis of stress variation at different sectors near the hole allows retrieving the real stress state. The developed method can significantly reduce errors in the determination of high residual stresses. The influence of materials properties, such as Young and plastic tangent moduli, Poisson ratio and yield stress, on the results obtained by the proposed procedure for plasticity error correction is investigated.
\end{abstract}

\section{Introduction}

Reliability and service life of materials are noticeably dependent on the residual stresses, which a high level can result in crack initiation, decrease of corrosion resistance, etc. Therefore, the development of reliable and informative methods of investigation of the stress state in structures is important. One of the most widely used methods for determination of residual stresses is the holedrilling method. The procedure involves drilling a small hole at the material surface and the strain is measured using a special strain gauge rosette attached concentrically around the drilled hole. According to the standard procedures [1], calculations are made under the assumption of linear elastic behavior of the material. It is widely known that a drilled hole might act as a stress concentrator that can further induce significant plastic deformation, particularly if the nearby residual stresses are high. It was previously shown that if the stress state does not exceed about $60 \%$ of the material's yield stress $\left(\sigma_{Y}\right)$ the errors induced by yielding around the hole are less than $3 \%$, but the plasticity error increases with increasing residual stresses [2-4]. This is associated with the fact that elastic deformations which for stresses should be calculated, are added to unknown plastic deformation. Various approaches have been proposed to calculate the plasticity effect on the results of residual stresses, mainly by using the material's yield strength [3]. However, determining the exact values of the yield stress require additional experimental efforts and may be a new source of error. The application of optical whole-field interferometry methods for determination of residual stresses allows gathering more information about the residual stress state 
of an object when compared with the data from the resistance strain gauges [5, 6]. This is achieved by calculating a significantly larger amount of displacement (or strain) values induced by the holedrilling. Electronic speckle-interferometry hole-drilling (ESPI-HD) method allows an automation of the data analysis and selection of predefined areas with displacements in the vicinity of the drilled hole in order to determine the residual stresses [6]. This additionally enables determination of the plasticity effect without the need of the material's yield stress [4].

\section{Determination of residual stresses by the ESPI-HD method}

Figure 1 schematically shows a typical ESPI setup [6] for surface displacement measurement. The light from a coherent laser source (1) is split into two parts using a beam splitter (2). Optical configuration for measuring in-plane displacements is based on the two-beam illumination arrangement. The observation direction is normal to the specimen surface. The two parts of the laser light interfere on the object's surface to form a speckle pattern, which can be imaged by a digital camera (7) and further calculations assume that the ESPI device measures only an in-plane component of the surface displacements.

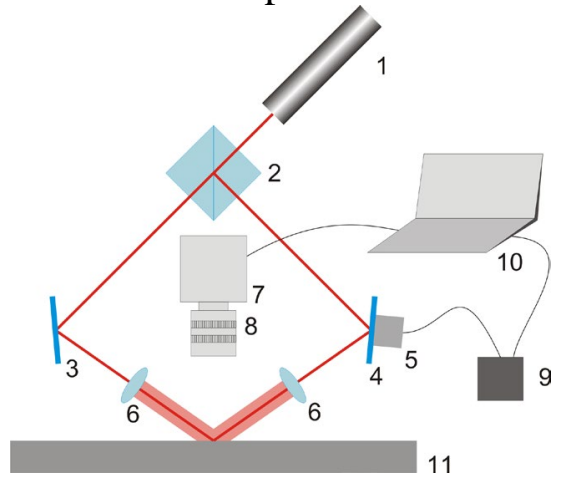

Fig. 1. A schematic presentation of the ESPI setup sensitive to in-plane displacement: 1 - laser, 2 - beam splitter, 3 mirror, 4, 5 - piezoelectric-driven mirror, 6 - beam expanders, 7, 8 -digital camera with lenses, 9 - piezo controller, 10 - computer, 11 - object.

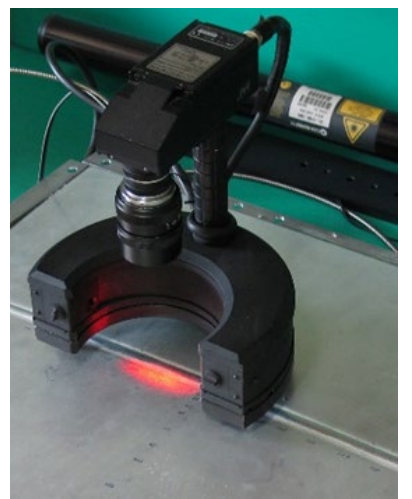

Fig. 2. The ESPI device for determination of residual stresses installed on a welded specimen

The displacements, caused by the relaxation of residual stresses in the bulk material, due to the drilling of blind holes are then measured using the ESPI method. Measurements are made in the following sequence [6]: the ESPI device is placed at the surface of the object and four-phase shifted speckle patterns are recorded, characterizing the initial state before drilling a hole. A phase map of the initial state is calculated by the four-step phase shifting algorithm. After stress relaxation caused by drilling a blind hole with a diameter of $0.5-2.0 \mathrm{~mm}\left(2 r_{0}\right)$, another set of speckle images, corresponding to the deformed state, is recorded and the resulting phase map is calculated. Computer processing of fringe patterns allows the evaluation of the displacements, $u_{x}$, in the laser illuminated area, as well as the calculation of the residual stresses. The proposed ESPIHD method uses displacements, $u_{x}(r, \theta)$, at a constant radius $r=2.5 r_{0}$ around a hole to calculate stresses because of: 1) the mean radius of the strain gauge rosette usually equals to $2.5 r_{0} ; 2$ ) automatic processing of speckle images is affected by the noise due to surface damage from chipping after drilling, as well as electronic noise from the digital camera, speckle-noise, laser radiation noise, etc. According to previous studies [4-6] the dependency of displacements, $u_{x}(r, \theta)$, due to blind hole drilling, on the residual stresses can be written in the following form [5, 6]:

$$
u_{x}(r, \theta)=F(r, \theta) \sigma_{x x}+G(r, \theta) \sigma_{y y}+H(r, \theta) \tau_{x y}
$$


where the basic functions $F(r, \theta), G(r, \theta)$ and $H(r, \theta)$ depend on the mechanical properties of the material, hole dimensions, radial position around the blind hole and can be determined from the displacement data of finite-element modeling of the blind hole drilling process $[5,6]$.

To find the three unknowns $\sigma_{x x}, \sigma_{y y}$ and $\tau_{x y}$ from a system of linear equations (1) it is sufficient to measure displacements $u_{x}$ at three points $u_{x, n}\left(2.5 r_{0}, \theta_{n}\right), n=1 \ldots 3$, which are located on a circle with a center at the drilling point [6]. The system of equations to be solved can adopt $n>3$ values $\theta_{n}$ to enable a least squares solution.

\section{Influence of the plasticity effect on residual stresses calculation}

Mathematical simulation with a finite element method was conducted in order to estimate the influence of plastic deformation on the accuracy of residual stress determination by the ESPI-HD method for different materials properties, such as Young $(E)$ and plastic tangent $(E t)$ moduli, Poisson ratio $(v)$ and yield stress $\left(\sigma_{Y}\right)$. FEM simulations were carried out by using the Code-Aster software. Due to the symmetrical geometry of the problem, only one quarter-plate finite element model consisting of 50976 HEXA8 elements was used. The following conditions were used in the simulation: hole diameter and depth of $1 \mathrm{~mm}$, object thickness was $5 \mathrm{~mm}$ and object width was $35 \mathrm{~mm}$. Stress-strain curves are presented in Fig. 3.

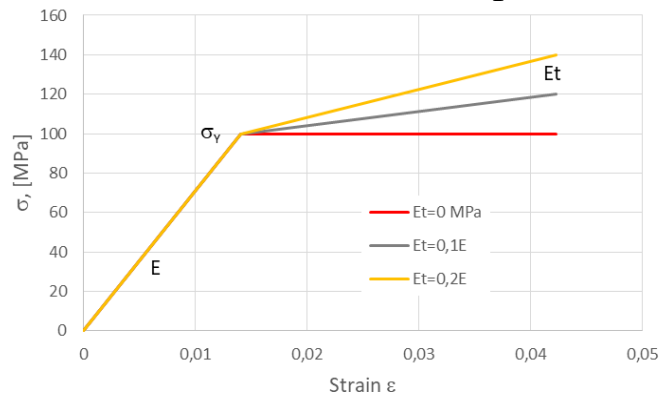

Fig. 3. Stress-strain curves used for FE simulation.

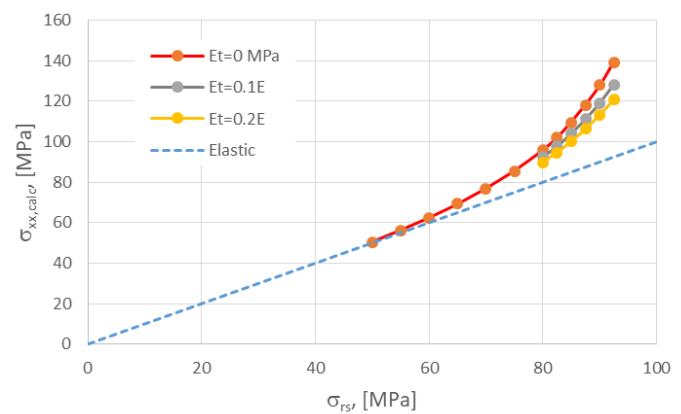

Fig. 4. Influence of plastic tangent module on residual stresses calculation. The dotted line represents an elastic material behavior.

As a result from simulation, displacement values of material surface were then calculated, depending on the stress level in the object. Afterwards, displacements, $u_{x}\left(2.5 r_{0}, \theta\right)$, were selected at different points at the surface located at a distance of $2.5 r_{0}$ from the hole center. Accordingly, the stress values $\sigma_{x x}, \sigma_{y y}$ and $\tau_{x y}$ were found using Eqs. 1. For simplicity in the simulations only the $\sigma_{x x}=\sigma_{r s}$ stress component was investigated while $\sigma_{y y}$ and $\tau_{x y}$ were assumed to be zero. The displacements $u_{x, \text { calc }}\left(2.5 r_{0}, \theta\right)$ at the distance of $2.5 r_{0}$ for angles of $\theta$ in the range [0;2 $\left.\pi\right]$ were calculated for a given $\sigma_{r s} / \sigma_{Y}$ ratio. Then, the system of equations (Eqs. 1) was composed and the values of $\sigma_{x x \text {,calc }}$ were calculated by the least-squares method. Then the residual stress determination error $\eta=\left(\sigma_{\text {calc }}-\sigma_{r s}\right) / \sigma_{r s}$, depending on the ratio $\left(\sigma_{r s} / \sigma_{Y}\right)$, was calculated. It is shown that for the ratio $\sigma_{r s} / \sigma_{Y}>0.6$, the difference between $\sigma_{r s}$ and $\sigma_{x x \text {,calc }}$ increases (see Fig.4).

Results of FE simulation for different sets of material properties $\left(E, E t, \sigma_{Y}, v\right)$ are presented in Table 1. It is possible to state that $\sigma_{Y}$ has the largest influence on the plasticity effect, while the Poisson ratio, $v$, does not affect the results significantly. Therefore an exact value of the yield stress is very important to achieve an accurate stress correction using the following steps of the procedure, proposed by Beghini et al, 2011 [3]: "1) evaluate the residual stress, by assuming the material as linear elastic ("as-elastic" residual stress); 2) calculate a plasticity effect intensity parameter; 3) re-evaluate the residual stress components from the as-elastic initially found and the plasticity effect intensity". Step 3 uses a relationship derived from FE simulation (Fig 4). 
Table 1. Influence of material properties on plasticity effect

\begin{tabular}{|r|r|r|r|r|r|r|r|r|r|r|}
\hline \multirow{4}{*}{$\mathrm{N}$} & \multicolumn{4}{|c|}{ input data } & \multicolumn{2}{c|}{$\begin{array}{c}\text { as-elastic } \\
\text { calculation }\end{array}$} & \multicolumn{2}{c|}{$\begin{array}{c}\text { plast.eff. correction } \\
\left(\sigma_{Y}=100 \mathrm{MPa}\right)\end{array}$} & \multicolumn{2}{c|}{$\begin{array}{c}\text { plast.eff. correction } \\
\left(\sigma_{Y}=110 \mathrm{MPa}\right)\end{array}$} \\
\cline { 2 - 12 } & $\begin{array}{r}\sigma_{r s}, \\
{[\mathrm{MPa}]}\end{array}$ & $\begin{array}{c}\sigma_{Y}, \\
{[\mathrm{MPa}]}\end{array}$ & $v$ & $E t$ & $\begin{array}{c}\sigma_{x x, \text { calc }} \\
{[\mathrm{MPa}]}\end{array}$ & $\eta,[\%]$ & $\begin{array}{c}\sigma_{x x, \text { calc }}, \\
{[\mathrm{MPa}]}\end{array}$ & $\eta,[\%]$ & $\begin{array}{c}\sigma_{x x, c a l c}, \\
{[\mathrm{MPa}]}\end{array}$ & $\eta,[\%]$ \\
\hline 1 & 85 & 100 & 0,33 & 0 & 109,45 & 28,8 & 85,0 & 0,0 & 89,70 & 5,5 \\
\hline 2 & 85 & 110 & 0,33 & 0 & 98,91 & 16,4 & 81,2 & $-4,4$ & 85,00 & 0,0 \\
\hline 3 & 90 & 100 & 0,33 & 0 & 127,93 & 42,1 & 89,9 & $-0,1$ & 95,43 & 6,0 \\
\hline 4 & 90 & 110 & 0,33 & 0 & 110,22 & 22,5 & 85,3 & $-5,2$ & 90,00 & 0,0 \\
\hline 5 & 85 & 100 & 0,27 & 0 & 110,38 & 29,9 & 85,3 & 0,4 & 90,06 & 6,0 \\
\hline 6 & 90 & 100 & 0,27 & 0 & 129,63 & 44,0 & 90,3 & 0,4 & 95,80 & 6,4 \\
\hline 7 & 85 & 100 & 0,30 & 0 & 110,17 & 29,6 & 85,3 & 0,3 & 89,98 & 5,9 \\
\hline 8 & 90 & 100 & 0,30 & 0 & 129,34 & 43,7 & 90,3 & 0,3 & 95,74 & 6,4 \\
\hline 9 & 85 & 100 & 0,33 & $0,1 \mathrm{E}$ & 103,87 & 22,2 & & & & \\
\hline 10 & 85 & 100 & 0,33 & $0,2 \mathrm{E}$ & 119,12 & 17,7 & & & & \\
\hline 11 & 90 & 100 & 0,33 & $0,1 \mathrm{E}$ & 100,05 & 32,4 & & & & \\
\hline 12 & 90 & 100 & 0,33 & $0,2 \mathrm{E}$ & 113,22 & 25,8 & & & & \\
\hline
\end{tabular}

\section{Determination of the plasticity effect by the ESPI-HD method}

There is no difference on the results for different sets of angles $\theta_{n}$, if the assumptions involved in basic theory, described in the ASTM E837 standard [1], are valid. But if the stress/strain relationship for the test material is nonlinear due to yielding or other causes, the calculated stresses may vary. One of the possibilities of the ESPI-HD method is the ability to compute stresses using the displacement data $u_{x}$ at points of an arc $B C$ (Fig. 5) that could be chosen around the drilled hole (Fig. 5). Variation of length (defined in radians by the angle $\psi$ ) and position (defined by the angle $\alpha$ ) of the circular arc $B C$ allows to develop a new technique for residual stress calculation, which takes into account the plastic deformation. The arc is defined by the following procedure. A point $A$ with polar coordinates $\left(2.5 r_{0}, \alpha\right)$ is marked on the circle. Regarding this point, the arc $B C$ that includes all available $N$ points with angular coordinates $\theta_{n}$ (where $\alpha-\psi \leq \theta_{n} \leq \alpha+\psi, n=$ $1, \ldots, N)$ is chosen. The displacements $u_{x}\left(\theta_{n}\right)$ on the arc $B C$ is then used to calculate the stress $\sigma_{x x, \text { calc }}(\alpha, \psi)$ from the system of Eqs 1 .

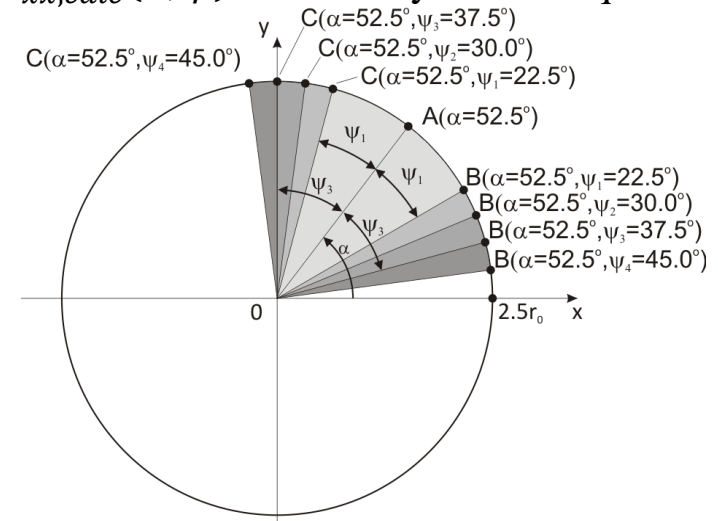

Fig. 5. Location of the arc $B C$ relative to the drilled hole

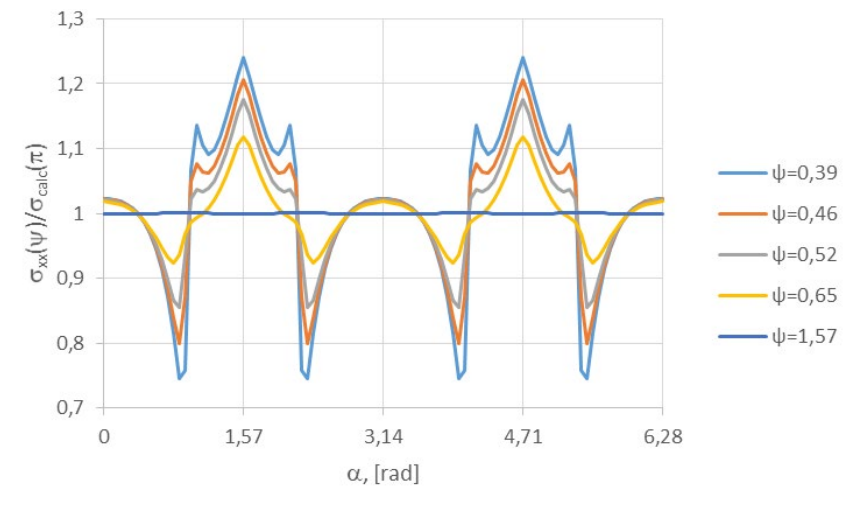

Fig. 6. Dependencies of the ratio, $\sigma_{x x, \text { calc }}(\alpha, \psi) /$ $\sigma_{x x, a v r}$ on $\alpha$ calculated by FEM for different values $\psi$ providing for $\sigma_{r s}=0.8 \sigma_{Y}$

The dependencies of the $\sigma_{x x, \text { calc }}(\alpha, \psi)$, calculated using displacement data measured on the arc $B C$ for an angle $\alpha$, are presented on Fig. 6 , for $\sigma_{r s}=0.8 \sigma_{Y}, E=70 G P a, E t=0 G P a$, and $v=0.33$. It is shown that in the presence of plastic deformation decreasing the length of the arc $B C$ (lower values of $\psi$ ) leads to an increase of the relative standard deviation $\left(R S D_{\psi}\right)$, where $R S D$ 
is defined as the ratio of the standard deviation of $\sigma_{x x, \text { calc }}(\alpha, \psi)$ to the averaged value $\sigma_{x x, \text { avr }}=$ $\sigma_{x x, \text { calc }}(\alpha, \pi)$ calculated using all points along the circle. The curves on Fig. 6 show a significant increase in the difference between the extreme and averaged value of the stress $\sigma_{x x, a v r}$ when the angle $\psi$ is decreased. This is a consequence of localized areas with plastic deformation. Thus $R S D_{\psi}$ is one of the parameters that may characterize the plasticity effect. Dependencies of the $R S D_{\psi}$ on $1 / \psi$ for different $\sigma_{r s} / \sigma_{Y}$ ratios are shown in Fig. 7a. It should be noted that $R S D_{\psi}$ tends to zero in the absence of plastic deformation. A monotonic increase of $R S D_{\psi}$ with increasing $\sigma_{r s} / \sigma_{Y}$ is observed. Thus, based on the analysis of the $R S D_{\psi}(1 / \psi)$ dependency, a proof about the absence or presence of plastic deformation after drilling a hole in the object is achieved. Fig. $7 \mathrm{~b}$ shows that the ratio $\sigma_{r s} / \sigma_{Y}$ is uniquely determined by the slope $k_{\psi}$ of the graph $R S D_{\psi}(1 / \psi)$.

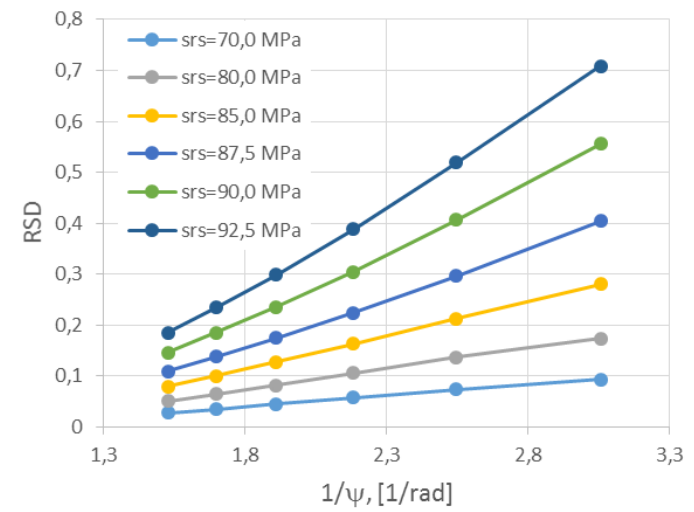

a)

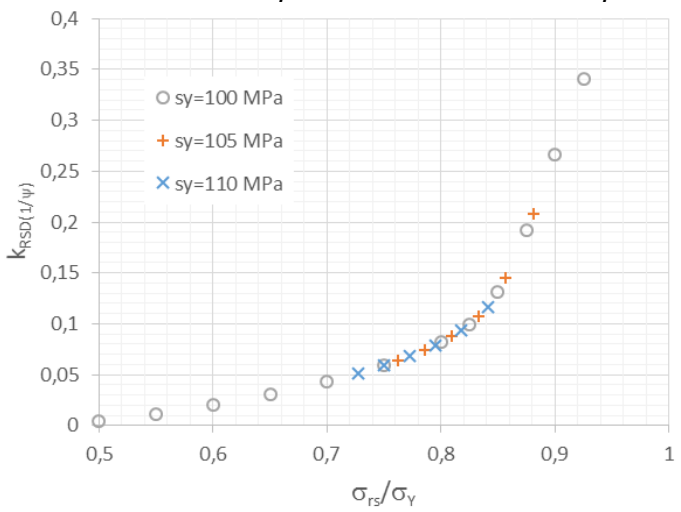

b)

Fig. 7. Dependencies of a) $R S D_{\psi}$ on $1 / \psi$ for different $\sigma_{r s} / \sigma_{Y}$ ratios, and b) $k_{\psi}$ on the ratio $\sigma_{r s} / \sigma_{Y}$ obtained by FE simulation ( $E=70 \mathrm{GPa}, E t=0 \mathrm{GPa}, v=0.33$ ).

The presently developed ESPI-HD method for determination of residual stresses taking into account the plasticity effects includes the following stages:

1. Find the functions $F\left(2.5 r_{0}, \theta\right), G\left(2.5 r_{0}, \theta\right)$ and $H\left(2.5 r_{0}, \theta\right)$ for real experimental conditions (diameter and depth of the hole, thickness of the object, mechanical properties of the material) using linear-elastic FE simulation or from a database.

2. Calculate averaged values of stresses $\sigma_{x x, a v r}$ and $\sigma_{y y, a v r}$ from Eqs. 1 using all displacement data along a circle with a radius of $2.5 r_{0}$ with the center in the drilling point. If $\sigma_{x x, a v r} \gg \sigma_{y y, a v r}$, do the next steps.

3. For a set of $\psi$ values in the range $[\pi / 6 ; \pi / 2]$ calculate stresses $\sigma_{x x, \text { calc }}(\alpha, \psi), \alpha=[0 ; 2 \pi]$.

4. Calculate $R S D_{\psi}$ for each value of $\psi$. Build the curve $R S D_{\psi}(1 / \psi)$ and determine the slope $k_{\psi, \exp }$ of the curve.

5. Compute the dependency of the slope $k_{\psi}$ on $\sigma_{r s} / \sigma_{Y}$ from elasto-plastic FE simulation.

6. Determine the plasticity effect by substituting the experimentally obtained $k_{\psi, \exp }$ value, calculated in Stage 4, into the dependency of $k_{\psi}$ on $\sigma_{r s} / \sigma_{Y}$ received obtained using FEM on Stage 5.

7. Calculate the residual stress applying the procedure described by Beghini et al, 2011 [3] and adapted to the displacement-stress form, but using experimentally determined plasticity effect.

The proposed procedure assumes that high residual stress exists only in one direction, which coincides with the laser illumination direction of the ESPI device. Other stress components are considered to be negligible. Such conditions are usual for residual stresses resulting from welding. As an example, residual stresses have been measured by the developed ESPI-HD method considering the plasticity effect in welded joints made of an aluminum alloy AlMg6 (Fig. 2). 


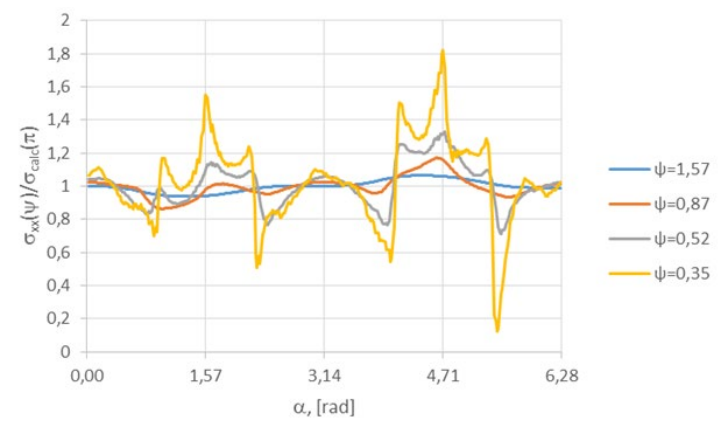

a)

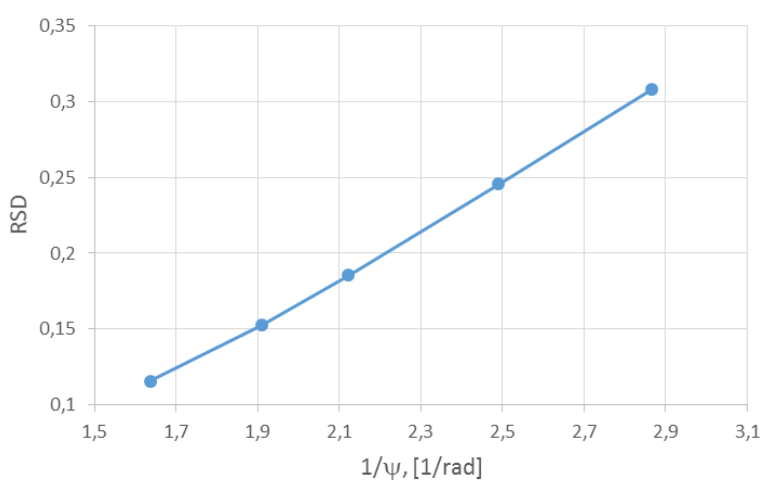

b)

Fig.8. Determination of residual stresses by the ESPI-HD method taking into account the plasticity effect: a) dependence of $\sigma_{x x, \text { calc }}(\alpha, \psi) / \sigma_{x x, \text { avr }}$ on $\alpha$ for different values of $\psi$; b) dependence of $R S D_{\psi}$ on $1 / \psi$. Material is AlMg6.

Approach, described in [6], determined that $\sigma_{x x, a v r}=246 \mathrm{MPa}$. Values of $\sigma_{x x, c a l c}(\alpha, \psi)$ and $R S D_{\psi}$ were calculated according to stages 2 to 4 and as a result the $R S D_{\psi}(1 / \psi)$ curve has been found (Fig. 8a and 8b). These graphs are similar to those obtained by mathematical modelling (see Fig. 6 and Fig. 7a) and they clearly show the presence of significant plastic deformation around the hole. FEM simulation has shown that the slope $k_{\psi}$ of the curve in Fig.8b fits values of the ratio $\sigma_{x x, a v r} / \sigma_{r s}=1.28$. Thus, in the drilling point the residual stresses are $\sigma_{x x}=192 \mathrm{MPa}$.

\section{Summary}

The proposed approach has been approved for measuring residual stresses taking into account the plasticity effect in a welded specimen with high uniaxial stress state. Determination of residual stresses in the welded plate shows that the theoretically predicted influence of high residual stresses on displacement variations is similar to those retrieved experimentally.

Application of the present approach for a biaxial stress state and incremental hole drilling should be further considered as well as experimental validations should be conducted.

\section{References}

[1] ASTM E837-13a, Standard test method for determining residual stresses by the hole-drilling strain-gage method, in Annual Book of ASTM Standards, Am. Soc. Test. Mat, 2013.

[2] Lin Y.C., Chou C.P., Error induced by local yielding around hole in hole drilling method for measuring residual stress of materials, J. Mater. Sci. Tech., Vol.11, 1995, pp. 600-604. https://doi.org/10.1179/mst.1995.11.6.600

[3] Beghini M., Santus C., Valentini E., Benincasa A., Experimental Verification of the Hole Drilling Plasticity Effect Correction, Materials Science Forum, Vol. 681 (2011), pp. 151-158. https://doi.org/10.4028/www.scientific.net/MSF.681.151

[4] Lobanov L., Savitsky V., Residual Stresses Determination with Plasticity Effects by Electron Speckle-Interferometry Hole-Drilling Method, Mat. Res. Proc., Vol. 2, 2017, pp. 389-394. https://doi.org/10.21741/9781945291173-66

[5] Baldi, A., A new analytical approach for hole drilling residual stress analysis by full field method, J. Eng. Mater. Technol., Vol. 127, No. 2, 2005, pp. 165-169.

https://doi.org/10.1115/1.1839211

[6] Lobanov L., Pivtorak V., Savitsky V. and Tkachuk G., Technology and Equipment for Determination of Residual Stresses in Welded Structures Based on the Application of Electron Speckle-Interferometry, Materials Science Forum, Vol. 768, 2014, pp. 166-173. 